MODELING, IDENTIFICATION AND CONTROL, 1982, vOL. 3, NO. 1, 41-51

doi:10.4173/mic.1982.14

\title{
A liquid cargo handling training simulator
}

\begin{abstract}
MALVIN VILLABØ†
Keywords: simulator, training, liquid cargo handling, network theory.

This paper describes a computer-based, real-time training simulator for liquid cargo handling. The mathematical model description is modular and emphasizes the flow generating part. The flow model applies network theory to describe the logical piping connections and the overall flow system performance, while Bernoulli's equation is used for the description of the piping elements. Numerically the Newton-Raphson method is applied for computation of the non-linear flow model. The programming language is HP-FORTRAN IV and the simulator is implemented on an HP minicomputer system. The instructor part of the system is interactive and controlled by the use of a standard ASCII terminal. The simulation part of the system is running in real-time or scaled real-time, and is controlled from the trainee's panel.
\end{abstract}

\section{Introduction}

This liquid cargo handling training simulator is concerned with crude oil handling aboard a typical crude oil carrier in a loading or an unloading situation. During these operations the deck officer of the watch is controlling several closely related systems. The following systems are incorporated in this simulator:

-the main cargo system

- the cargo stripping system

- the ballast system

-the crude oil washing system

Monitoring of the most important ship parameters is also incorporated.

The simulator is an operational training simulator and does not pay any attention to maintenance and repair caused by maloperations. The performance of the simulator coincides with the requested operations and the status of malfunctions. By this approach the trainees can detect the operational results of malfunctions and maloperations, and they can counteract the malfunctions by the use of possible redundancies in the systems.

Formerly, liquid cargo handling simulators were of interest mainly because of maloperations and resulting costly maintenance and repair of limited system components. During later years, liquid cargo handling systems have become more comprehensive. The systems have been improved and new systems have been added due to safety reasons and international regulations. The required understanding of the overall system performance is thus growing at the same time as proper operation of the components in the cargo handling systems is important.

This simulation considers these changing requirements. The simulator can be used to teach operational personnel in operating a typical system configuration. The simulator can also be used as an operational research tool. By emphasizing the overall

† The Ship Research Institute of Norway, Department of Marine Engineering. 
system performance, profit can be attained from optimization of the total loading/ unloading procedures.

\section{System description}

The liquid cargo handling part of the simulator can schematically be drawn as indicated in Fig. 1. In Fig. 1 we can recognize several physical systems which in the proceedings will be treated as separate items.

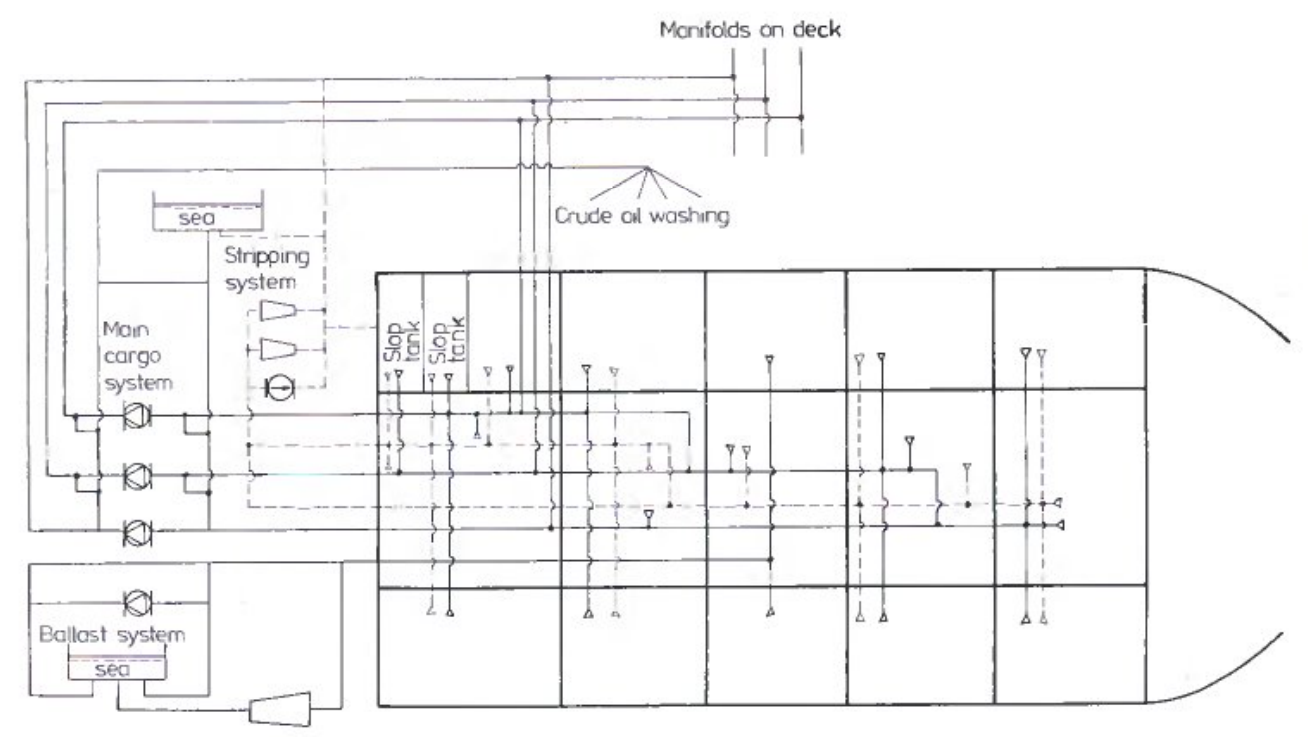

Figure 1. Layout of the piping systems.

The main cargo system consists of three centrifugal pumps each with a performance of approx. 1.5 MW and a piping network. The piping network has a connection to each of the cargo tanks, to the two slop tanks, and to the recipient ashore via manifolds on deck.

The cargo stripping system consists of two ejector pumps, a reciprocating pump, and a piping network. The two ejectors are driven by cargo oil from the discharge cross-over in the main cargo system. The piping network has a connection to each of the cargo tanks, to the two slop tanks, to the sea, and to the manifolds on deck.

The ballast system consists of a centrifugal pump and an eductor driven by the centrifugal pump. A piping network is connecting two segregated ballast tanks (starboard and port wing tank no. 3), sloptank no. 1, and the sea to the pumping facilities.

The crude oil washing (COW) system is connected to the discharge cross-over at the main cargo pumps. The COW system consists of a piping network distributing crude oil to tankcleaning equipment in each of the cargo oil tanks.

All the flow generating systems, the main cargo system, the cargo stripping system, the ballast system, and the COW system, are connected to the tanks aboard the ship and the tanks thus constitute the information integrating part of the system. 
The simulator considers 5 centre tanks, 5 port wing tanks, 5 starboard wing tanks, and 2 slop tanks. Port and starboard wing tanks no. 3 are segregated ballast tanks and thus connected only to the ballast system. The rest of the tanks are connected to scveral flow generating systems as indicated in Fig. 1.

In addition to the strict liquid cargo handling related part, a module considering the behaviour of the ship is also incorporated in the simulator. This module is concerned with list, trim and draught conditions.

\section{Mathematical modeling}

From the system description it can be realized that the flow generating systems can be treated more or less independent of each other. This argument is considered when defining the system boundaries, and we end up with some rather decoupled mathematical models

- the flow models

- the crude oil washing model

-the tank model

- the ship condition model

\subsection{Flow models}

The main cargo system, the cargo stripping system, and the ballast system can be mathematically modelled by sticking to the same mathematical approach. In the following only one of these systems is therefore discussed more in detail. The flow systems are described mathematically by using network theory. The network theory is applied to describe all the possible physical connections and the redundancies in the systems, and the approach is found to be convenient. Additionally every piping element in the network is described by non-linear pressure-flow rate relations.

When starting with the physical system descriptions in Fig. 1, each of the flow generating systems can be represented by a directional network, where each link is representing one or more physical piping elements in between two neighbouring nodes (branching points), see Fig. 2. This network can be investigated by applying some well known theorems from the network theory and a network tree can be established.

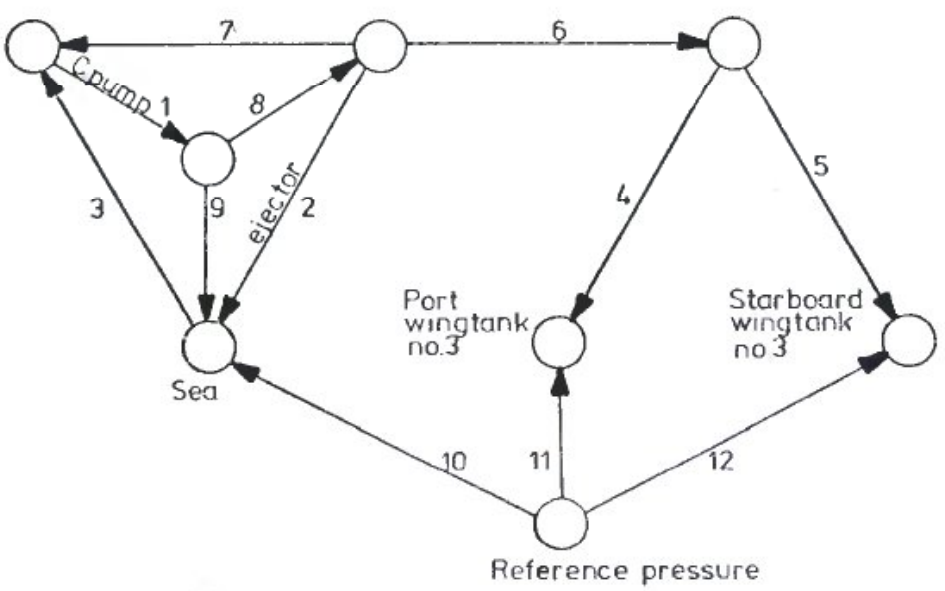

Figure 2. Network of the ballast system. 
Every link in the network, not contained in the tree, is further on defined as meshdefining links and given the identification code $i, i \in(1, \mathrm{NMESH})$ where:

$$
\mathrm{NMESH}=\mathrm{NLINK}-\mathrm{NNODE}+1
$$

NMESH-total number of meshes (loops)

NLINK - total number of links

NNODE-total number of nodes

NMESH meshes can now be defined in the network where each mesh consists of only one mesh-defining link and several tree links. The definition of all these meshes can be described mathematically by a matrix, $A$, where:

$a_{i j}=0$ - link no. $j$ is not incorporated in mesh no. $i$

$a_{i j}=1$-link no. $j$ is included in mesh no. $i$, and the direction coincides with the mesh direction

$a_{i j}=-1-$ link no. $j$ is included in mesh no. $i$, directed opposite of the mesh direction

The dimension of the matrix $A$ is $(\mathrm{NMESH} \times \mathrm{NLINK})$ and it contains all necessary information about the configuration of the piping system. The matrix describing the ballast system (see Fig. 2) looks like:

$$
A=\left[\begin{array}{rrrrrrrrrrrr}
1 & 0 & 0 & 0 & 0 & 0 & 1 & 1 & 0 & 0 & 0 & 0 \\
0 & 1 & 0 & 0 & 0 & 0 & 0 & 1 & -1 & 0 & 0 & 0 \\
0 & 0 & 1 & 0 & 0 & 0 & -1 & -1 & 1 & 0 & 0 & 0 \\
0 & 0 & 0 & 1 & 0 & 1 & 0 & 1 & -1 & 1 & -1 & 0 \\
0 & 0 & 0 & 0 & 1 & 1 & 0 & 1 & -1 & 1 & 0 & -1
\end{array}\right]
$$

In the following the network will be related to a flow system, and two well known physical relations will be applied

- the pressure around a mesh adds up to zero

-mass conservation in every node

\section{Pressure relation}

From the definition of the matrix $A$ each row describes a mesh; which links are incorporated in the actual mesh and their direction. This knowledge together with the pressure law can mathematically be described as:

$$
A \boldsymbol{p}=0
$$

where:

$$
\boldsymbol{p}=\left(p_{1}, p_{2}, \ldots, P_{\mathrm{NLINK}}\right)^{T}-\text { vector of all link pressures }
$$

\section{Flow rates}

When studying the matrix $A$, every column describes a link; which meshes are passing through it and their respective directions. The flow rate in every link is therefore a linear combination of the flow rate in the mesh-defining links. Mathematically the expression will look like:

$$
q=A^{T} q_{m}
$$


where:

$q=\left(q_{1}, q_{2}, \ldots, q_{\mathrm{NLINK}}\right)^{T}$-flow rates in all the links

$\boldsymbol{q}_{m}=\left(q_{m, 1}, \ldots, q_{m, \mathrm{NMESH}}\right)^{T}$-subvector of $q$ containing flow rates in the meshdefining links

\section{Link descriptions}

Equations (2)-(3) describe the mathematical features of the network as such, but there is still a shortage of NLINK equations to make the mathematical flow system description consistent. These missing NLINK equations can be established by the pressure drop-flow rate relation of each link in the system. These equations will in general be non-linear and in this simulator we have used the following simplification

where

$$
p_{i}=K_{i, 0}+K_{i} q_{i}\left|q_{i}\right|, \quad i=1,2, \ldots, \text { NLINK }
$$

$p_{i}=$ pressure increase along link no. $i$

$K=$ coefficients describing the piping elements contained in link no. $i$

This simplification does still consider the main contributions to the pressure-flow rate relations, which are static pressure drop due to geodetic levels and pressure drop due to friction. The descriptions of the flow generating systems do not consider the power plant for generating pump rpm. The system boundaries are well defined taking pump rpms, list and trim angles, and states of the remote controlled valves as input for dynamic modification of the coefficients in eqn. (4).

The rpm of the pumps are modelled as:

$$
\dot{n}_{i}=\frac{n_{i, \text { ref }}-n_{i}}{T}, \quad T \text {-time constant }
$$

\subsection{The crude oil washing system}

The crude oil washing (COW) system is making use of crude oil from the discharge cross-over at the main cargo pumps and distributing the oil via tankcleaning equipment back to the cargo tanks. The simulator does not consider the cleaning efficiency. The COW system can therefore be considered as leakages in the main cargo system sending crude oil back to the tanks, and a separate comprehensive flow model for this system, influencing the unloading performance, is avoided. The leakage in the main cargo system caused by the COW system is represented as a conventional link in the actual network with the following mathematical description:

where

$$
p=K_{W o}+K_{W} N^{-2} q_{L}^{2}
$$

$$
\begin{aligned}
& N \text {-total number of tankcleaning equipments operated } \\
& q_{L} \text {-total leakage rate of flow back to the tanks via the COW system }
\end{aligned}
$$

The flow rate of oil back to each tank because of the washing operations is related to the relative number of tankcleaning equipments operated in each particular tank

$$
q_{\mathrm{cow}, j}=\frac{N_{j}}{N} q_{L}
$$


where:

$q_{\text {cow }, j}$ flow rate of oil back to tank no. $j$

$N_{j}-$ number of tankcleaning equipments operated in tank no. $j$

\subsection{The tank model}

The content of each tank is integrated when all the flows influencing the tanks are known.

$$
\dot{V}_{j}=\sum \text { input rate of flows }-\sum \text { output rate of flows }
$$

The cross-section of each tank is in general level dependent. The levels of content are therefore calculated from the contents in order to make sure that the mass conservation requirement is fulfilled.

$$
s_{j}=f_{j}\left(V_{j}\right)
$$

where:

$$
\begin{aligned}
& V_{5} \text {-oil volume in tank no. } j \\
& s_{j} \text {-oil level in tank no. } j
\end{aligned}
$$

In the flow models the pressure-flow rate representation of the tanks looks like:

$$
p_{j}=\rho g s_{j}+p_{\text {ing }}
$$

where:

$p_{\text {ing }}$-inert gas pressure

The simulator does not consider the inert gas system and the tank ventilating system. The tank model is, however, fitted for these extensions by already incorporating a dummy inert gas pressure.

\subsection{Ship conditions}

Only the most important parameters for description of the ship conditions are incorporated in the simulator. These are the trim angle, the list angle, and the draught. All these parameters are found from the light mass, the amount of cargo, the cargo distribution, and the geometry of the hull. This is a rather slowly varying process in relationship to the rest of the simulator incorporated processes, and only static calculations are considered.

$$
\left.\begin{array}{l}
m=m_{1}+\sum_{\text {tanks }} m_{j} \\
x_{c}=\left(m_{1} x_{1}+\sum_{\text {tanks }} m_{j} x_{j}\right) / m \\
y_{c}=\left(m_{1} y_{1}+\sum_{\text {tanks }} m_{j} y_{j}\right) / m
\end{array}\right\}
$$


At rest, the force of buoyancy acting upwards must be equal to the total mass of the ship, and the centre of buoyancy must be in the same vertical line as the centre of gravity.

where:

$$
\left.\begin{array}{c}
\theta=f_{\theta}\left(m, x_{c}\right) \\
\Phi=f_{\Phi}\left(m, y_{c}\right) \\
z_{d f}=f_{d f}\left(m, x_{c}, y_{c}, \theta\right) \\
z_{d a}=f_{d a}\left(m, x_{c}, y_{c}, \theta\right)
\end{array}\right\}
$$

$$
\theta \text {-trim angle }
$$

$\Phi$ _ list angle

$z_{d f}$-draught forward

$z_{d a}$-draught aft

$m$-total mass of the ship

$m_{1}$-light mass of the ship (incorporating stores, fuel, etc.)

$m_{j}$-mass of cargo in tank no. $j$

$x_{c}, y_{c}-$ centre of gravity

\section{Implementation}

The mathematical models described here are successfully implemented on a Hewlett-Packard minicomputer at NSFI, The Ship Research Institute of Norway. The models are implemented as FORTRAN programmes and they make use of a general purpose real-time simulation software package formerly implemented on the HP computer. The software configuration is indicated in Fig. 3.

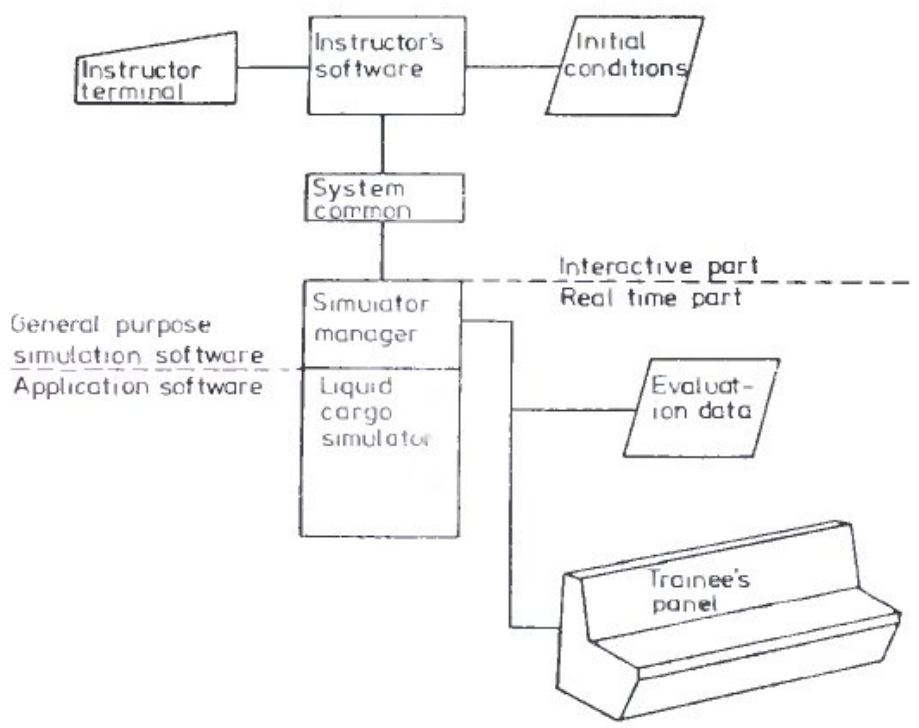

Figure 3. System configuration. 


\section{Flow models}

The flow models are the main consumers of computer resources. These models are non-linear and the numerical algorithms thus include iterating procedures. The non-linear set of equations looks like:

$$
A \cdot \boldsymbol{p}\left(\boldsymbol{q}_{m}\right)=F\left(\boldsymbol{q}_{m}\right)=F\left(\boldsymbol{q}_{m, 0}\right)+\left.\frac{\partial F}{\partial \boldsymbol{q}_{m}}\right|_{\boldsymbol{q}_{m}=\boldsymbol{q}_{m}, 0}\left(\boldsymbol{q}_{m}-\boldsymbol{q}_{m, 0}\right)=0
$$

By using the Taylor expansion of the non-linear equation, the iteractive procedure will become:

$$
\boldsymbol{q}_{m, n+1}=\boldsymbol{q}_{m, n}-\boldsymbol{z}
$$

where:

$$
\begin{aligned}
D \boldsymbol{z} & =\boldsymbol{F}\left(\boldsymbol{q}_{m, n}\right) \\
D & =\left.\frac{\partial F}{\partial \boldsymbol{q}_{m}}\right|_{\boldsymbol{q}_{m}=\boldsymbol{q}_{m}, n}
\end{aligned}
$$

$D$-matrix describing the linearized system $(\mathrm{NMESH} \times \mathrm{NMESH})$

$n$-number of iterations

Under normal conditions only one or two iterations should be sufficient when using the last sample as initial conditions. Iterating is generally ended when the relative corrections, $S_{i}=z_{i} / q_{m, i}$ come below a predefined value.

The matrix $D$ can be found analytically expressed as a function of $\boldsymbol{q}_{m}$ when accepting the simplification assumed in eqn. (5).

Because of the comprehensive computational task and because of the possibility of singularities occurring in the matrix $D$, the dimensions of the flow systems in operation are dynamically investigated and kept at a minimum. There are no reasons for calculating the flow in links, which are closed because of the actual state of the control valves.

\section{Pressure}

When the rate of flow in each link is known, the pressure increase in each link can be calculated by using eqn. (4). Additionally the pressure in a node is chosen as a reference pressure and the absolute pressure in all the other nodes can be computed.

\section{Integration}

The tank model is integrated by implementing the model as difference equations. Difference equations are also used in order to describe the rpm of the pumps.

\section{Alarms}

Two types of alarms are generated. The first type is related to maloperations of components and malfunctions in the systems. Typical examples here are cavitation in the centrifugal pumps and valves stuck in a former position. The second type of alarms is directed towards illegal operations and they are related to the training philosophy. The COW system is here a typical example. The physical performance of the COW system is of minor interest in relationship to the consequences of washing a tank with a crude oil quality different from what that particular tank initially contained. 


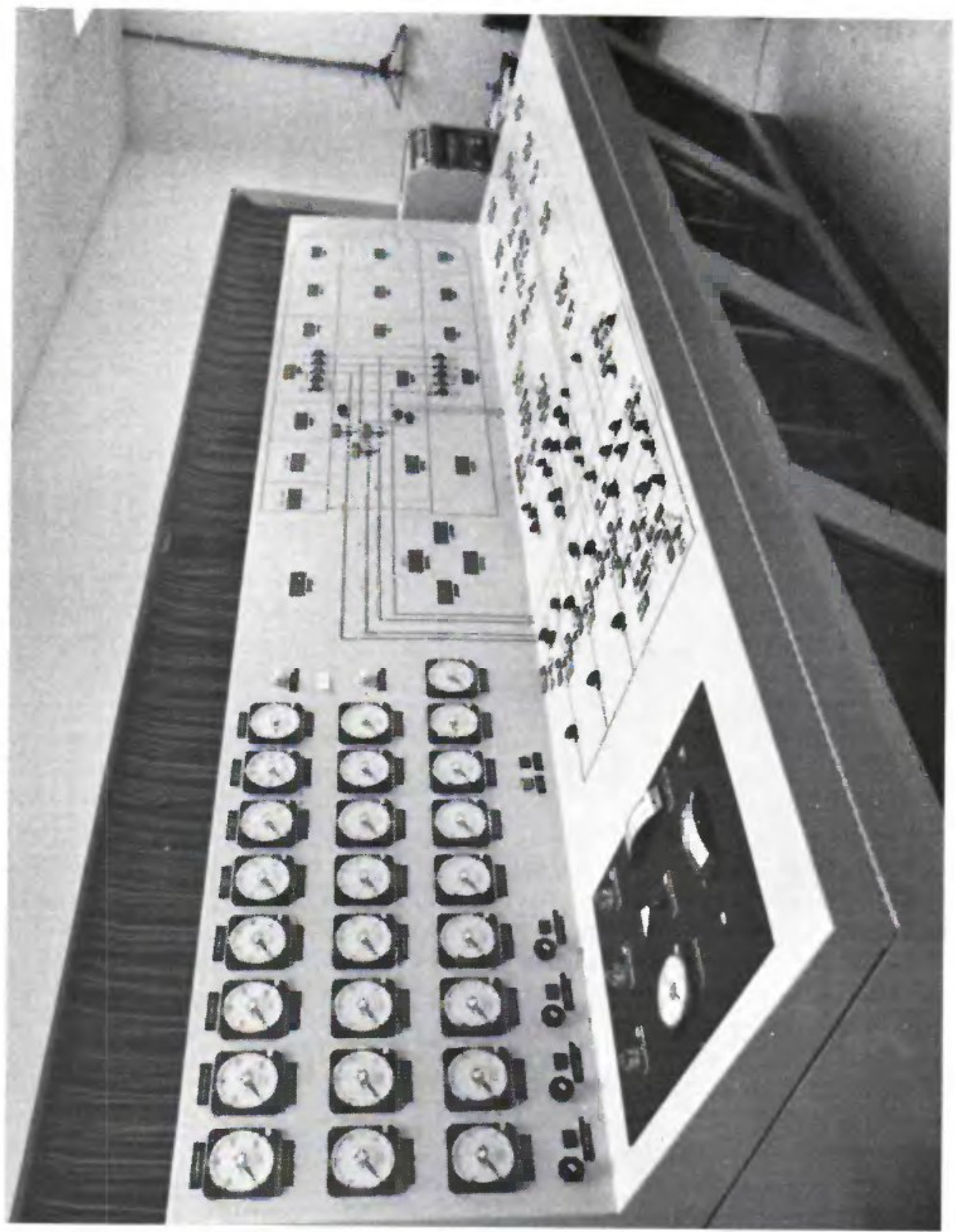

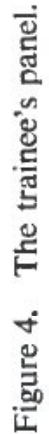




\section{Operation}

The simulator will be operated from the trainee's panel which looks like a conventional control panel in the cargo control room, see Fig. 4. From this panel all remote controllable valves and pumps in the incorporated systems can be operated. Pump manufacturers as well as experienced deck officers have contributed to the functional requirements and the pressure, flow, and alarm responses are similar to what is experienced in real life.

\section{Training philosophy}

There are two main objectives in the training philosophy. The trainees shall learn how to operate properly the systems and the subsystems under normal conditions and when malfunctions occur, e.g. consequences of trottling, changing rpm, etc. The trainees shall also experience the results from changing operational procedures at the component level, e.g. pumps operated in parallel or series, etc.

The second main training objective is directed towards the overall process performance. The hourly expenses for a crude oil carrier are considerable, and time saving is often tantamount to money saving. Often the charter contains also financial claims for exceeding the prefixed time for loading/unloading operations. The simulator can in this respect be used for operational investigations. How can the time consumption be minimized when all the equipment still operates within the specifications?

\section{Training}

Most of the exercises will concentrate on normal operational conditions in order to familiarize the trainees with the system and to make them understand all the available possibilities for loading/unloading (routeing in the piping networks). A predefined set of relevant malfunctions is available. During exercises single malfunctions as well as combinations of malfunctions can be initiated and the trainees will learn how to cope efficiently with them.

For training evaluation after or during exercises, time series of vital parameters from the simulation are available on secondary memory. These parameters can be plotted as a function of time or as a function of each other. In case evaluation is preferred during exercises, the simulator can be preliminarily stopped and the situation frozen. The cargo handling process varies rather slowly. To increase the efficiency of the training, the simulation can be performed at speeds faster than in real life.

\section{Conclusion}

This paper describes the realization of a liquid cargo handling simulator which can be used both for training of operational personnel and for operational research. From a mathematical point of view, the flow models constitute the most interesting part where the application of network theory seems to be convenient both in this particular solution and in future applications. This mathematical approach, as well as the simpler mathematical models of the other subsystems, are discussed and related to the simulator requirements. The level of mathematical sophistication in a training simulator is of minor importance in relationship to the practical application. Familiarization to this kind of educational equipment and to the training programme are just as important. 


\section{REFERENCES}

Jeppson, R. W. (1976). Analysis of flow in pipe networks (Ann Arbour Science Pub. Inc., Michigan).

PotT, R. B., and Oliver, R. M. (1972). Flows in transportation networks (Academic Press, New York).

VILlabø, M. (1979). Documentation pump simulator. Internal report 239.51080.00.03 (NSFI, Trondheim, Norway); (1980). Software for cargo oil pumping simulator. Commission report 230.55229.00.01 (NSFI, Trondheim, Norway). 